\title{
Impacto de la Manufactura Esbelta, Manufactura Sustentable y Mejora Continua en la Eficiencia Operacional y Responsabilidad Ambiental en México
}

\author{
Carlos Monge ${ }^{(1)}$, Jesús Cruz $^{(2)}$ y Fabián López ${ }^{(2)}$ \\ (1) Universidad Autónoma de Nuevo León, Escuela de Graduados en Administración e Ingeniería Industrial. \\ Guerrero y Progreso, Colonia Treviño, 64570 Monterrey-México (e-mail: carlosmongep@prodigy.net.mx) \\ (2) Universidad Autónoma de Nuevo León, Centro de Desarrollo Empresarial y Posgrado, Facultad de \\ Contaduría Pública y Administración. Pedro de Alba s/n, San Nicolás de los Garza-México \\ (e-mail: jesusphd@prodigy.net.mx; fabian.lopezpz@gmail.com)
}

Recibido Ene. 03, 2013; Aceptado Feb. 18, 2013; Versión final recibida Abr. 10, 2013

\begin{abstract}
Resumen
Se ha desarrollado un modelo matemático para relacionar la manufactura esbelta, la manufactura sustentable y la mejora continua con la eficiencia operacional y responsabilidad ambiental en la industria manufacturera en una región de México. El modelo fue desarrollado empleando la técnica de ecuaciones estructurales mediante mínimos cuadrados parciales y se demostró su capacidad predictiva. El modelo muestra también que los factores estudiados afectan los resultados financieros, la satisfacción de empleados, la cultura de sustentabilidad y el impacto medioambiental en plantas de manufactura discreta de Apodaca en México. El estudio establece las bases para análisis posteriores del modelo con el fin de predecir desempeños en la eficiencia operacional y sus variables clave, así como prescribir estrategias y tácticas para la adopción exitosa de los enfoques analizados. Esto permitirá lograr ventajas competitivas en calidad, costos, tiempo de entrega, flexibilidad e innovación.
\end{abstract}

\section{Impact of Lean Manufacturing, Sustainable Manufacturing and Continuous Improvement on Operational Efficiency and Environmental Responsibility in Mexico}

\begin{abstract}
A mathematical model to relate lean manufacturing, sustainable manufacturing and continuous improvement with the operational efficiency and environmental responsibility in the manufacturing industry in a region of México was developed. The model was established using structural equations including partial least squares and demonstrated its predictive capability. The model also shows that the factors studied affect financial results, employee satisfaction, sustainable culture and environmental impact in manufacturing plants of Apodaca in Mexico. The study also lays the foundation for further analysis of the model, for predicting performance in operational efficiency and its key variables and for prescribing strategies and tactics for successful adoption of the approaches discussed. This will allow achieving competitive advantages in quality, cost, delivery time, flexibility and innovation.
\end{abstract}

Keywords: lean manufacturing, sustainable manufacturing, continuous improvement, operational efficiency, environmental responsibility 


\section{INTRODUCCIÓN}

El término manufactura esbelta (lean) fue introducido en 1990 en occidente con la publicación del libro la "Máquina que cambió al mundo" del Dr. James P. Womack (Womack et al., 1990) basado en el estudio de cinco años del Sistema de Producción Toyota realizado por el MIT dentro de su Programa Internacional de Vehículos de Motor (IMVP) por sus siglas en inglés. El libro mostraba cómo la administración, los trabajadores de línea y los proveedores podían trabajar conjuntamente en las plantas de manufactura, para mejorar sustancialmente la eficiencia operacional a través de: Mejoras de la calidad y de las entregas a tiempo, reducción de costos, velocidad de entrega, tiempos de ciclo, incremento de la productividad por empleado y mejora de la capacidad de la planta sin incremento de personal, impactando positivamente los resultados financieros (Cooper et al., 2008), resultados similares pueden obtenerse en otros contextos demandantes en conocimiento, como el sector del cuidado de la salud con la aplicación de la cultura esbelta (Toussaint et al., 2013), el enfoque esbelto (lean) requiere nuevos hábitos y nuevas habilidades, el concepto esbelto no es un destino sino un viaje, que implica una búsqueda insaciable de la mejora, por lo cual la filosofía esbelta y la manufactura esbelta están estrechamente ligadas con la mejora continua, y ambas con la mejora del desempeño operacional (Toussaint et al., 2013; Murugesan et al., 2012; Vinohd et al., 2012; Koenigsaecker, 2009; Cooper et al., 2008), por su parte Murugesan et al., (2012) y Vinohd et al., (2012) indican que el logro de una mayor eficiencia operacional lograda a través de la manufactura esbelta tiene una influencia significativa, positiva y directa en el logro de ventajas competitivas del negocio como son: calidad a la primera intención, reducción de tiempos de entrega e incremento de la productividad (Ghosh, 2013).

En relación a la manufactura sustentable (Jiang et al., 2012; Millar et al., 2011) el segundo componente considerado en esta investigación, estudios recientes de (MIT y BCG, 2013, 2011, 2009) y Bergmiller et al., (2011) , así como los de Millar et al., (2011), Murugesan et al., (2012), Wills, (2009a, 2009b) y (EPA, 2000) muestran que la manufactura sustentable puede ayudar a las organizaciones a lograr mejor desempeño operacional y mejora en el desempeño ambiental, apoyando los esfuerzos de sustentabilidad de las plantas e impactando directa y positivamente los: Resultados financieros (RF), impacto de la huella de carbono (IHC), cultura de sustentabilidad de la organización (CSO) y satisfacción de los empleados (SE), el tema es muy importante si se considera que la manufactura a nivel global consume grandes cantidades de recursos y genera enormes cantidades de desperdicios, baste decir que la manufactura a nivel mundial consume un tercio de la energía mundial y genera el $36 \%$ del total del $\mathrm{CO}_{2}$ (Millar et al., 2011) el principal gas de efecto de invernadero (GEI), en México Irastorza et al., (2010) indican que la industria generadora de energía contribuye con un $21 \%$ y la manufactura en un $8 \%$ a la emisión del mencionado gas, sin embargo es la industria quien más energía consume contabilizando $60 \%$, mientras que solo el $30 \%$ de las empresas en México cuentan con un sistema de medición energética, lo que manifiesta la escasa conciencia ambiental de las empresas (Rodríguez, 2011), es por lo tanto, fundamental que las plantas de manufactura adopten prácticas sustentables (Bergmiller et al., 2011) o manufactura sustentable, aunque esta última expresión tenga diferentes significados para diferentes personas como lo consigna Millar et al., (2011), en este sentido hay evidencias que la sustentabilidad y la manufactura sustentable han están cobrando creciente relevancia a nivel mundial como lo revelan los estudios de: Schneider Study, (2011) y los del MIT y BCG, (2013, 2011, 2009), que manifiestan tendencias en los últimos años hacia un cambio en la actitud de los altos ejecutivos de las organizaciones, de considerar que el tema de la sustentabilidad y su adopción, son importantes solo si traen beneficios económicos de corto plazo, a considerar la sustentabilidad un importante factor de diferenciación (Millar et al., 2011) para el logro de ventajas competitivas y estratégicas por la vía de la innovación de productos y procesos, que permiten acceder a nuevos mercados, conseguir una mejora de la imagen de la organización ante la sociedad, reputación de la marca e incremento del valor para los accionistas. En el caso de México un estudio parecido fue conducido por la compañía consultora KPMG (KPMG, 2009) y los resultados obtenidos son similares a los estudios realizados por el MIT y BCG ya citados.

Con respecto a la mejora continua, Murugesan et al., (2012), Koenigsaecker, (2009) e Imai, (1986) refieren que la mejora continua (kaizen), el tercer componente del presente estudio, es un ingrediente fundamental para la ventaja competitiva, sobrevivencia y crecimiento de las organizaciones, sin embargo la literatura revela que la manufactura esbelta y la mejora continua no han sido bien comprendidas en occidente, y como consecuencia se considera que su implantación consiste únicamente en aplicar herramientas/técnicas esbeltas de manera aislada, y no como un proceso de mejora continua, generándose de esta manera "silos" esbeltos y no una implantación integral que traería grandes beneficios y ventajas competitivas, de acuerdo a Bergmiller et al., (2011) y Liker et al., (2011) esto se debe fundamentalmente a la carencia de compromiso

y liderazgo de la alta administración en las plantas occidentales, lo mismo ocurre en otros contextos como el de cuidados de la salud, donde un requisito fundamental de la mejora continua, es la actitud hacia esta en todos los niveles de la organización, desde la alta administración hasta los trabajadores en la línea de 
acción, y requiere también un verdadero cambio cultural que modifique la forma cómo trabaja una organización (Toussaint et al., 2013; Shook, 2010). En el contexto de la manufactura y en particular en las plantas de México, la situación no es muy diferente en cuanto a la adopción de la mejora continua, la manufactura esbelta y sustentable, éstas iniciativas no se reflejan en acciones de mejora continua según lo revela un estudio realizado por Reyes-Aguilar, (2002) y que se traduce en la escasa participación de las plantas mexicanas en estos enfoques ya sea de manera grupal o individual a través de las propuestas individuales de mejora (PIMs), en estas plantas el personal confunde la administración por objetivos o el trabajo cotidiano con la mejora continua, en algunos otros casos se realizan esfuerzos aislados como proyectos seis sigma, y en algunas plantas no existe en absoluto orientación sistemática, disciplinada y comprometida de la organización hacia la mejora continua.

Ahora bien, aunque se menciona con frecuencia en la literatura los efectos positivos que la manufactura esbelta, sustentable y la mejora continua tienen en el desempeño operacional, existen pocos estudios empíricos realizados, particularmente en países en desarrollo, empleando estadística descriptiva (Millar et al., 2011; Murugesan et al., 2012), modelos de ecuaciones estructurales (Lee, 2012; Vinohd et al., 2012) o bien alguna otra técnica de análisis multivariado como regresión múltiple, análisis factorial y componentes principales (Murugesan et al., 2012) que vinculen a la manufactura esbelta, sustentable y la mejora continua con la eficiencia operacional y la ventaja competitiva, entendidas estas como un desempeño sobresaliente en: costo, calidad, velocidad y flexibilidad. Es importante mencionar que los estudios empíricos existentes, analizan los aspectos mencionados de manera separada y no de forma simultánea, como propone la presente investigación empírica, lo cual constituye una diferencia significativa y valiosa.

Por otra parte, se reconoce hoy día que la manufactura debe jugar un rol importante en la búsqueda de la sustentabilidad debido a la gran cantidad de recursos que consume y los desperdicios que genera, en este sentido es importante mencionar que es posible obtener mayor eficiencia en la producción como resultado de la aplicación de la manufactura esbelta, y que esto trae consigo menos consumo de energía, materias primas por unidad, reducción de emisiones al aire y al agua, así como menor generación de residuos sólidos y/o peligrosos y disminución del riesgo de incumplimiento de las regulaciones gubernamentales, es pues determinante la adopción por parte de las plantas de manufactura de iniciativas sustentables (Millar et al., 2011), que conduzcan a una mejora de su eficiencia operacional y que les brinde ventajas competitivas, particularmente hoy día, en un entorno globalizado y sumamente competido. Un ingrediente de presión extra para las plantas de manufactura, es la creciente conciencia medioambiental de la sociedad y las cada vez más exigentes regulaciones o los compromisos gubernamentales en la materia (Millar et al., 2011), son justamente estos aspectos mencionados los que inspiran el desarrollo de la presente investigación.

Finalmente el modelo resultante del presente estudio puede ser empleado como guía por las plantas de manufactura de Apodaca, México para orientar acciones de mejora en la eficiencia operacional y la responsabilidad medioambiental de manera combinada, con el fin de impactar la triple línea final, es decir utilidades-sociedad-medioambiente (Slaper, 2011). Por otra parte, dadas sus características predictivas, el presente estudio también persigue, establecer los cimientos para que partiendo del modelo propuesto y usando información obtenida del mismo, puedan realizarse investigaciones posteriores con técnicas multivariadas complementarias, con el fin de predecir situaciones futuras en el desempeño operacional y sus variables clave, lo que permitiría prescribir estrategias y tácticas exitosas a las plantas de manufactura de Apodaca, México, para lograr de ventajas competitivas, en calidad, costos, tiempos de entrega, flexibilidad, mejora continua, innovación, medio ambiente, así como en seguridad y moral de los empleados, en este sentido en la literatura es posible encontrar un estudio (Arrieta et al., 2010) con la misma intención aunque con un enfoque diferente.

\section{REVISIÓN DE LA LITERATURA}

Para la investigación se efectuó una revisión de la literatura existente relacionada con el tema de estudio con el fin de conocer las aportaciones que se han hecho acerca del mismo, los elementos teóricos encontrados proceden de diversas fuentes bibliográficas reconocidas como: Revistas indexadas, libros y bases de datos digitales entre ellas: Scielo México, Scielo Chile, Información Tecnológica Chile, bases de datos de la Universidad Nacional Autónoma de México (UNAM), Academic One file, EBSCO Host Academic Search Complete, EBSCO Business Source Complete, EBSCO Econlit with full text, Springer y Elsevier. La literatura encontrada se relaciona con el planteamiento del problema de investigación y sirvieron para

extraer las variables dependientes e independientes, así como para definir la manera de operacionalizarlas e identificar los ítems que constituyen el instrumento de medición (escala). Cabe mencionar asimismo, que la revisión de la literatura mostró las brechas de conocimiento existentes en la literatura actual acerca de los temas tratados en el presente estudio y permitió la justificación y orientación de la investigación. 


\section{Manufactura esbelta}

El término esbelto (lean) que da inicio a la manufactura esbelta fue introducido por el Dr. James $P$. Womack en 1990 en occidente con la publicación del libro la Máquina que cambió al mundo (Womack et al., 1990) basado en el estudio de cinco años del Sistema de Producción Toyota realizado por el MIT dentro de su Programa Internacional de Vehículos de Motor (IMVP) por sus siglas en inglés, el concepto esbelto y la manufactura esbelta persiguen mejoras sustanciales del desempeño operacional o como Marugesan et al., (2012) refiere ventajas competitivas como: Calidad, costo, precio, velocidad en la entrega, consistencia en la entrega, innovación y flexibilidad (mejor, más barato, más rápido y más ágil), esto es posible a través de la identificación y eliminación continua y sistemática de los desperdicios (actividades que no agregan valor) o "mudas" (término japonés de desperdicios), con el activo involucramiento de todos los empleados de una organización en proyectos de mejora continua (Imai, 1986). La eliminación de las "mudas" como: sobreproducción, esperas, transporte, procesos innecesarios, inventario, movimientos y defectos (Cottyn et al., 2011; Imai, 1986) se realiza a través de 5 principios rectores del pensamiento esbelto que son: a) definición de valor desde la perspectiva del Cliente, b) mapeo de los procesos de producción y de servicios, c) crear flujo en los diferentes procesos, d) jalar la producción y e) búsqueda de la perfección a través de la mejora continua (Cottyn et al., 2011). La aplicación disciplinada, comprometida y eficazmente lideradas de estos principios eventualmente conduce a las plantas hacia la conversión en empresas esbeltas y a la obtención de enormes beneficios en términos de eficiencia operacional y ventajas competitivas (Ghosh, 2013; Lee, 2012; Murugesan et al., 2012; Vinohd et al., 2012).

En relación con la manufactura esbelta y el pensamiento esbelto, existen varios estudios en la literatura (Amin et at., 2013; Austin et al., 2013; Ghosh, 2013; Lee et al., 2012; Vinodh et al., 2012; Murugesan et al, 2012) e incluso algunos de ellos realizados en contextos latinoamericanos (Cardozo et al., 2011; Millar et al., 2011; Arrieta et al., 2010; Pérez et al., 2011), en el ámbito mexicano desafortunadamente pocos estudios relevantes fueron encontrados (Reyes-Aguilar, 2002). Enseguida se comentará con mayor detalle los alcances de cada uno de los estudios citados al inicio de este párrafo, en Amin et al., (2013), se desarrolló un modelo matemático de optimización con el fin de ayudar a las plantas de manufactura a seleccionar las estrategias esbeltas (lean) adecuadas para eliminar o reducir los desperdicios dadas las restricciones de los recursos de planta y de esta manera mejorar el desempeño operacional, en dicho modelo se evalúa cuantitativamente el valor percibido de las estrategias lean en la reducción de los desperdicios "muda" a través de un solución óptima construida con MATLAB, los resultados muestran que el valor percibido de la reducción de "muda" puede cambiar significativamente de acuerdo a las políticas y estrategias de producto consideradas por el fabricante.

Por su parte Austin et al., (2013) refiere que dadas la necesidades cambiantes del Cliente y los requerimientos tecnológicos actuales, las organizaciones se ven forzadas a implantar iniciativas "lean" en sus procesos de producción con el fin de conseguir mejorar el desempeño operacional (eficiencia operacional) y lograr ventajas competitivas, el estudio presenta en el contexto automotriz cuna del concepto esbelto, un modelo desarrollado empleando la técnica de Análisis Jerárquico de Procesos (AHP) por sus siglas en inglés, en su implantación se aplicó un cuestionario a 15 compañías automotrices de la India, el modelo relaciona las prácticas y técnicas esbeltas con los objetivos competitivos e identifica, cuáles de las diferentes herramientas esbeltas tienen mayor impacto en el logro de un desempeño esbelto. Con Ghosh, (2013) el estudio muestra la situación actual en la implantación de prácticas esbeltas en plantas de manufactura de la India y su impacto en el desempeño operacional, el estudio se realizó aplicando un cuestionario a 79 plantas en las diferentes regiones de India, y dado que la manufactura esbelta es un constructo multidimensional se encontró que el $80 \%$ de las plantas ha implantado varias dimensiones de la manufactura esbelta como son: enfocarse en las necesidades del Cliente, sistemas de producción jalada, reducción de tiempo de intercambio de moldes (SMED), mantenimiento productivo total (TPM), relación con proveedores, control estadístico de procesos y solución incluyente de problemas. Se encontró asimismo que se ha incrementado la productividad, la calidad a la primera intención, reducido el tiempo de entrega y los inventarios así como el espacio ocupado, a manera de síntesis, los encuestados afirmaron que la calidad a la primera intención, reducción del tiempo de entrega y el incremento de la productividad son los tres principales impulsores de la adopción de la manufactura esbelta en aquel contexto. Vinodh et al., (2012) describe que con frecuencia se considera que la manufactura esbelta mejora la competitividad de los

negocios, sin embargo, existe poca evidencia en la literatura de estudios empíricos que validen el impacto positivo en el desempeño organizacional, con este propósito en este estudio se obtuvieron datos empíricos para medir las prácticas de la manufactura esbelta que prevalecen en las industrias en India, fue desarrollado un modelo de ecuaciones estructurales para validar las mediciones e hipótesis y los resultados muestran como la manufactura esbelta está correlacionada con el mejoramiento del desempeño operacional de las organizaciones, este estudio tiene un enfoque similar al de la presente investigación, debido a que 
usa modelación con ecuaciones estructurales para determinar el impacto directo de la manufactura esbelta con la eficiencia operacional.

Murugesan et al., (2012) presenta un estudio que contempla un modelo de regresión múltiple para correlacionar dos variables independientes, la manufactura de clase mundial (WCM) por sus siglas en inglés y las iniciativas esbeltas (lean) o manufactura esbelta con la variable dependiente ventaja competitiva o desempeño operacional, todas estas variables son multidimensionales, los datos empíricos fueron obtenidos de 125 plantas de manufactura del sur de la India y el resultado mostró que tanto la manufactura de clase mundial y la manufactura esbelta están estrechamente ligadas con el desempeño operacional y la ventaja competitiva, lo cual fue revelado por un coeficiente de determinación $\mathrm{R}^{2}$ de 0.83 , es importante mencionar que Upadhye et al., (2010) considera que la manufactura de clase mundial (WCM) y la manufactura esbelta son lo mismo. Lee, (2012) en un estudio realizado con 970 compañías de manufactura en 18 países, aplicó modelación con ecuaciones estructurales para probar empíricamente la relación entre la manufactura esbelta y la administración total de la calidad (TQM) por sus siglas en inglés en el desempeño operacional (eficiencia operacional), los resultados revelaron que las dos aspectos tienen un impacto positivo, directo y estadísticamente significante en el desempeño operacional medido a través de las dimensiones: calidad, costo velocidad y flexibilidad, en algunos otros estudios estas dimensiones son expandidas para incluir algunas otras mediciones (Murugesan et al., 2012), en el contexto latinoamericano se han realizado algunos estudios en relación con la adopción de la manufactura esbelta, Cardozo et al., (2011) analizaron el proceso productivo de 45 empresas pequeñas y medianas productoras de queso en Venezuela, para determinar la adopción de las dimensiones de la manufactura esbelta en particular de las $5 S$ y la adopción de prácticas sustentables (manufactura sustentable) en estas organizaciones, los resultados muestran que las empresas tienen limitaciones en la adopción de ambos enfoques, limitando su competitividad. Arrieta et al., (2012) también muestran en su estudio una difusión muy baja de las iniciativas esbeltas en el sector de la confección en las empresas no extranjeras en Colombia, el caso de México no es muy diferente de acuerdo al estudio de Reyes-Aguilar, (2002).

\section{Manufactura sustentable}

La manufactura sustentable (Jiang et al., 2012; Millar et al., 2011) basada en principios de sustentabilidad y desarrollo sustentable, a diferencia de la manufactura esbelta que busca en un proceso de mejora continua reducir o eliminar desperdicios o "mudas", persigue eliminar los desperdicios ambientales en los procesos productivos que de acuerdo a Wills, (2009a, 2009b) son: Energía (particularmente la eléctrica), agua, emisiones al aire, emisiones al agua, uso irracional de materiales, residuos sólidos y/o peligrosos, transportación y daño a la biodiversidad, mejorando así el desempeño operacional y ventajas competitivas. Bergmiller et al., (2011) en otro estudio refiere que las plantas de manufactura exitosas en la aplicación de la manufactura esbelta, deberían también ser exitosas al implantar principios sustentables y manufactura sustentable, su estudio realizado en plantas de manufactura finalistas del premio Shingo, que es un reconocimiento a la excelencia en la manufactura en plantas de Canadá, EEUU y México, explora la sinergia existente entre la manufactura esbelta y la sustentabilidad, de esta forma se establece la relación entre la manufactura esbelta y la sustentable, Cardozo et al., (2011) en un estudio reciente en el contexto latinoamericano realizado en 45 plantas PYME's productoras de queso de Venezuela para determinar la adopción de prácticas esbeltas y sustentables, refiere que la manufactura esbelta y la manufactura sustentable están vinculadas, y que la falta de adopción de estas provoca una pérdida de la posición competitiva.

Queda establecido entonces, que la manufactura esbelta ayuda al medio ambiente aun inadvertidamente, ahora bien, la prevención de la contaminación y la preocupación por el medio ambiente (Kidwell, 2006), conducen a una mejora en la eficiencia operacional, que en esencia es lo que persigue la manufactura esbelta dada la liga entre estos dos enfoques de manufactura, de hecho ambas filosofías tienen varios puntos de coincidencia y comparten algunas herramientas como son: Mapeo de la corriente de valor o VSM por sus siglas en inglés (Toussaint et al., 2013) y $5 S$ con algunas modificaciones menores, así por ejemplo, el VSM utilizado en la manufactura esbelta para identificar y eliminar los desperdicios "mudas" consignados por el Sistema de Producción de Toyota (TPS) por sus siglas en inglés, puede ser usado también para

identificar los desperdicios ambientales, y como una herramienta de administración sustentable (Silveira et al., 2009) que facilita la generación de proyectos de mejora continua verdes (Wills, 2009a; EPA, 2000). En el contexto de la zona del Caribe Millar et al., (2011) desarrollaron un estudio descriptivo para determinar el nivel de adopción de la manufactura sustentable en los países de esa región encontrando que las plantas del Caribe poseen un escaso conocimiento de la manufactura sustentable y la implantación de estas iniciativas es escasa, en México la situación es similar en la adopción de la manufactura sustentable por parte de las empresas, esto puede deberse a la poca cultura de sustentabilidad en la industria de la manufactura, que no les permite ver que el compromiso medioambiental apoya el logro de beneficios 
económicos (Kidwell, 2006) y simultáneamente mejora la huella ecológica (Slaper, 2011), asimismo, no son capaces de identificar en la combinación de la manufactura esbelta, sustentable y mejora continua la oportunidad de conseguir ventajas competitivas y estratégicas, crear cultura de sustentabilidad y mejorar la calidad, costos, tiempos de entrega, imagen en la comunidad, generar mayor valor para los accionistas y satisfacción de los empleados (MIT y BCG, 2013, 2011, 2009; KPMG, 2009).

\section{Mejora continua}

La mejora continua (Imai, 1986), aplicada a la manufactura, es en esencia una filosofía que involucra de manera individual y a través de propuestas individuales de mejora (PIMs) o grupal a través de proyectos de mejora (PM), círculos de calidad, grupos pequeños o proyectos seis-sigma, a todo el personal de una organización, desde la alta administración, hasta los empleados de línea en la búsqueda interminable de niveles superiores en materia de: Calidad, costos, tiempos de entrega, flexibilidad, seguridad y moral de los empleados (Koenigsaecker, 2009), la característica primordial es que los esfuerzos de mejora pueden ser pequeños (Kaizen), dramáticos (Innovación) o la combinación de ambos, siempre y cuando dichos esfuerzos sean continuos (Imai, 1986), y es determinante para el éxito de la mejora continua, la activa, motivada y copiosa participación de los empleados. En cuanto a la mejora continua, los estudios citados en las secciones de manufactura esbelta y manufactura sustentables refieren la realización de acciones de mejora continua (Austin et al., 2013; Ghosh. 2013; Jiang et al., 2012; Lee et al., 2012; Murugesan et al, 2012; Cardozo et al., 2011; Wills, 2009a; EPA, 2000), esto es consistente ya que tanto las iniciativas de manufactura esbelta y sustentable son enfoques de aplicación continua y no puntuales (Bergmiller et al., 2011) condición fundamental para el éxito de ambas iniciativas. Por otra parte ya fue comentado en las secciones anteriores el impacto de las iniciativas esbeltas y sustentables en el desempeño operacional $u$ eficiencia operacional, de hecho la manufactura esbelta y la sustentable con frecuencia son consideradas filosofías de mejora continua (Murugesan et al., 2013; Toussaint et al., 2013; Bergmiller et al., 2011; Cardozo et al., 2011). Silveira et al., (2009) incluso menciona que la técnica VSM empleada en la manufactura sustentable puede ser usada simultáneamente para identificar los desperdicios ambientales y como una herramienta de administración sustentable (Silveira et al., 2009) que facilita la generación de proyectos de mejora continua verdes para eliminarlos (Wills, 2009a; EPA, 2000), la condición determinante para el éxito de la mejora continua es el involucramiento y apoyos decididos de la alta administración, así como la ejecución de las actividades de mejora de manera sostenida (Bergmiller et al., 2011, Liker et al., 2011), abundante y motivada del personal de manera individual a través de propuestas individuales o grupal por medio de proyectos de mejora interdisciplinarios (Imai, 1986).

En este proceso, se identificaron también las brechas de conocimiento de los elementos teóricos estudiados, mismas que cimentan la dirección de la investigación y la aportación al avance del conocimiento del presente estudio, encontrándose que dicha brecha consiste en que los estudios previos analizados consideran los impactos de la manufactura esbelta, sustentable y la mejora continua en el desempeño operacional de manera aislada y no integrada con una medición a través de un índice de eficiencia operacional y responsabilidad ambiental como lo propone el presente estudio. También debe mencionarse como parte de la brecha el hecho de que los estudios encontrados en la literatura fueron realizados en contextos diferentes al de Apodaca, México.

\section{DESCRIPCIÓN DE LAS HIPÓTESIS}

Ho. Existe un grado de impacto positivo, directo y estadísticamente significante similar de las variables: Efectividad de la manufactura esbelta (EME), efectividad de la manufactura sustentable (EMS) y efectividad de la mejora continua (EMC) en el índice de eficiencia operacional y responsabilidad ambiental (IEORA), que a su vez influencia positivamente en similar grado a los: Resultados financieros (RF), satisfacción de empleados (SE), impacto en la huella de carbono (IHC) y cultura de sustentabilidad de las organizaciones (CSO) lo que se establece en las ecuaciones 1 y 2.

\footnotetext{
Ho: (EME, EMS, EMC) $\rightarrow \quad$ IEORA

$\beta_{\mathrm{EME}, \mathrm{IEORA}} \approx \beta_{\mathrm{EMS}, \mathrm{IEORA}} \approx \beta_{\mathrm{EMC}, \mathrm{IEORA}}$

Ho: IEORA $\rightarrow$ (RF, IHC, SE, CSO)

$\lambda_{\mathrm{RF}, \mathrm{IEORA}} \approx \lambda_{\mathrm{IHC}, \mathrm{IEORA}} \approx \lambda_{\mathrm{SE}, \mathrm{IEORA}} \approx \lambda_{\mathrm{CSO}, \mathrm{IEORA}}$
}

Ha1. Existe una diferencia significativa en el nivel de impacto positivo de las variables: EME, EMS y EMC en el IEORA, que se expresa en la ecuación 3. 
Ha1: $\beta_{\text {EME,IEORA }} \neq \beta_{\text {EMS,IEORA }} \neq \beta_{\text {EMC,IEORA }} \rightarrow$ IEORA

Ha2: El nivel de impacto positivo de la variable EME en el IEORA es mayor que ell de las variables EMS y EMC, esta declaración se expresa en la ecuación 4.

Ha2: $\beta_{\text {EME,IEORA }}>\left(\beta_{\text {EMS,IEORA, }} \beta_{\text {EMC,IEORA }} \rightarrow\right.$ IEORA

Ha3: El nivel de influencia positiva del IEORA en las variables RF, IHC, SE y CSO es diferente lo que se indica en la ecuación 5.

Ha3: $\lambda_{\mathrm{RF}, \mathrm{IEORA}} \neq \lambda_{\text {IHC,IEORA }} \neq \lambda_{\mathrm{SE}, \mathrm{IEORA}} \neq \lambda_{\mathrm{CSO}, \text { IEORA }}$

Ha4: El nivel de influencia de la variable RF en el IEORA es mayor que el de las variables IHC, SE y CSO, lo que indica en la ecuación 6 .

Ha4: $\lambda_{\text {RF,IEORA }}>\left(\lambda_{\text {IHC,IEORA }}, \lambda_{\text {SE,IEORA }}, \lambda_{\text {CSO,IEORA }}\right)$

\section{MODELO GRÁFICO}

El modelo gráfico o modelo conceptual del estudio está constituido por cuatro variables latentes, una de ellas es de naturaleza exógena, tres variables endógenas EMS, EMC e IEORA, tres indicadores exógenos y trece endógenos.

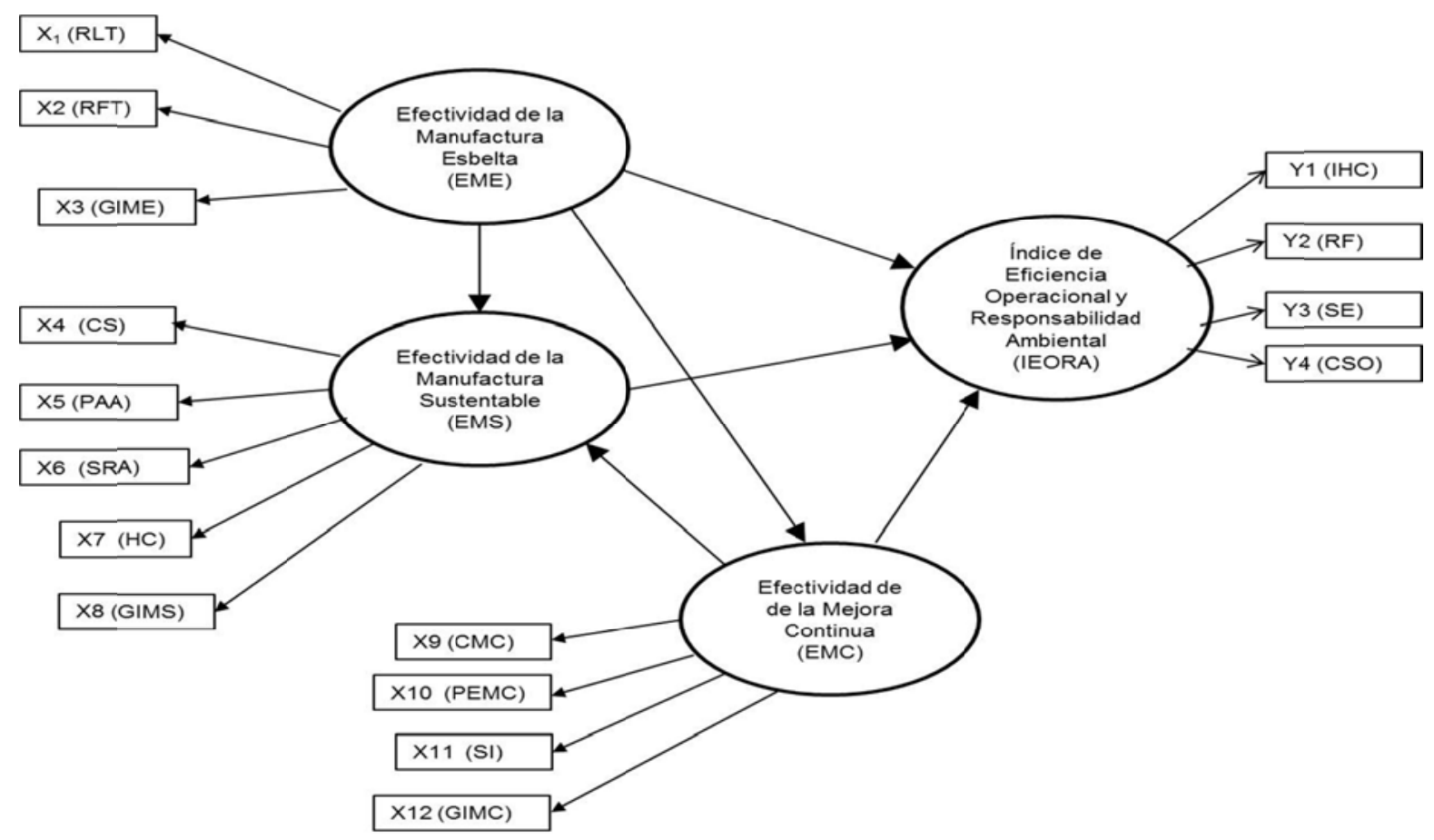

Fig. 1: Modelo gráfico

Fuente: Propia.

\section{DESCRIPCIÓN DE VARIABLES E INDICADORES}

Variable latente exógena: Efectividad de la manufactura esbelta (EME). Variables latentes endógenas: Efectividad de la manufactura sustentable (EMS), efectividad de la mejora continua (EMC), índice de eficiencia operacional y responsabilidad ambiental (IEORA). Indicadores exógenos: Reducción del tiempo de entrega (RLT), reducción del tiempo de flujo (RFT), grado de implantación de la manufactura esbelta (GIME). Indicadores endógenos: Compromiso con la sustentabilidad (CS), producción de productos y servicios amigables al ambiente (PAA), superación de las regulaciones ambientales (SRA), huella de carbono (HC), grado de implantación de la manufactura sustentable (GIMS), compromiso de la administración con la mejora continua $(\mathrm{CMC})$, participación de los empleados en la mejora continua (PEMC), sugerencias implantadas (SI), grado de implantación de la mejora continua (GIMC), impacto en la huella de carbono (IHC), resultados financieros (RF), satisfacción de los empleados (SE), cultura de sustentabilidad de la organización (CSO). 


\section{ESTUDIO DE CAMPO}

\section{Tipo de investigación}

El presente estudio es de tipo exploratorio, descriptivo, correlacional y explicativo de los impactos de las variables: EME, EMS, EMC y IEORA en indicadores de desempeño clave de las plantas de manufactura discreta y repetitiva de Apodaca, México como son: RF, SE. IHC y CSO.

\section{Diseño y técnica de la investigación}

La técnica de investigación utilizada es no experimental, empleando técnicas documentales, bibliográficas y con estudio de campo vía encuesta aplicada en forma directa o por correo electrónico, empleando un instrumento de medición diseñado exprofeso con 38 ítems y considerando como unidad de análisis a los gerentes de planta y/o gerentes de operaciones. Por la naturaleza de los constructos latentes involucrados, las interrelaciones entre los constructos independientes y dependientes, la existencia de más de un constructo dependiente, además de la intención predictiva de la investigación, se emplea para la modelación estadística, la técnica de modelación mediante ecuaciones estructurales y el empleo de mínimos cuadrados parciales (PLS-SEM) por sus siglas en inglés, se prefirió esta técnica a la basada en covarianza (CB-SEM) por sus siglas en inglés, debido a que PLS-SEM no supone normalidad de los datos, no condiciona el tamaño mínimo de la muestra además que, mientras CB-SEM es adecuada para la confirmación de relaciones estructurales, PLS-SEM es más apropiada para la predicción y/o generación de teorías (Hair et al., 2011).

\section{Elaboración del instrumento (escala)}

El instrumento de medición se basó en escalas nuevas debido a que en la revisión de la literatura no fue posible identificar estudios relacionando todos los aspectos discutidos en la investigación, sin embargo y cuando fue posible fueron utilizadas mediciones validadas que han sido aplicadas en estudios previos, la escala está constituida por 38 ítems distribuidos en cuatro secciones, cada sección para cada una de las variables latentes. El cuestionario fue revisado por investigadores académicos con experiencia en el diseño de instrumentos de medición y posteriormente por expertos en manufactura esbelta y sustentable, el resultado de este proceso fue la reducción del número de ítems originalmente en 47 y modificaciones a la forma de estructurar los ítems, los ítems solicitan respuestas en escala Likert de 1-5 (1-nada y 5-totalmente) o datos duros para medir con mayor efectividad los aspectos asociados a: EME, EMS, EMC e IORA.

Dadas las diferencias de escalas empleadas en el instrumento de medición, las respuestas a los ítems fueron estandarizadas a valores típicos de " $z$ ", restándole al valor del ítem para cada caso la media de la muestra y el resultado dividiéndolo entre la desviación estándar de la misma muestra, asimismo, previo a la alimentación al modelo PLS-SEM se ponderaron. El instrumento fue validado y refinado en una segunda fase en una prueba piloto con 10 casos, y para medir la validez y consistencia interna se utilizó la técnica de alfa de Cronbach seleccionando únicamente los ítems por constructo con un valor no menor a 0.70 (Tavakol et al., 2011), la validez y confiabilidad de la escala y de los constructos fue realizada en la ejecución del modelo PLS-SEM y se discuten en la sección de resultados. En la tabla 1 se muestran los ítems de la escala por constructo e indicadores correspondientes, así también se indican entre paréntesis el tipo de respuesta que debe ser proporcionada.

\section{Selección de la muestra}

Para el establecimiento de la muestra, se hicieron las siguientes consideraciones: Población: Las plantas de manufactura discreta y repetitiva medianas y grandes de Apodaca, NL, que de acuerdo a la base de datos del 2012 de la Cámara de la Industria de la Transformación del estado de Nuevo León (CAINTRA NL) son 60. Muestra: Grupo de 38 plantas determinadas vía fórmula con valores de p y q de 0.5 y para un porcentaje de error de $5 \%$ de acuerdo a Hernández-Sampieri et al., (2010). El instrumento de medición fue enviado a los gerentes de planta u operaciones de las 60 plantas, se recibieron únicamente $40(66.66 \%)$ respuestas productivas del total, mismas que constituyen la muestra final de la investigación. Con relación al tamaño de la muestra, los modelos de PLS-SEM no son restrictivos en el tamaño, y es frecuente encontrar menciones en la literatura de estudios de ecuaciones estructurales, mediante PLS-SEM relacionadas con la cantidad suficiente de casos a usar en PLS-SEM (Hair et al., 2012; Hair et al., 2011; Henseler et al., 2009), estas menciones sugieren que para el caso de PLS-SEM con escalas reflexivas, la cantidad de casos debe ser igual a 10 veces el número de trayectorias estructurales entrantes al constructo con mayor cantidad de trayectorias, en el caso de esta investigación el constructo con mayor cantidad de trayectorias entrantes es IEORA con 3 lo que sugiere 30 casos como apropiado. Cabe mencionar que la literatura también refiere estudios PLS-SEM con resultados razonables y con una cantidad de casos menor (20 o 10 casos) a la "regla de dedo" referida (Henseler et al, 2009). En última instancia la determinación de si el tamaño de la 
muestra es adecuado, y ayuda a obtener resultados razonables y estadísticamente significativos, se obtiene a través del cumplimiento de los criterios de calidad para el diagnóstico de modelos PLS-SEM (Hair et al., 2012), en consecuencia, los resultados obtenidos pueden ser extrapolados a otros municipios de México.

Tabla 1. Ítems de la escala

\begin{tabular}{|c|c|c|}
\hline CONSTRUCTO & INDICADOR & ITEM \\
\hline EME & $\begin{array}{l}\text { RFT } \\
\text { RLT }\end{array}$ & $\begin{array}{l}\text { 1) Mapeo de procesos (porciento implantado) } \\
\text { 2) } 5 S \text { (porciento implantado) } \\
\text { 3) Administración visual y controles visuales (porciento implantado) } \\
\text { 4) Intercambio rápido de moldes (porciento implantado) } \\
\text { 5) Calidad a la primera y dispositivos a prueba de errores (porciento implantado) } \\
\text { 6) Conteos cíclicos (porciento implantado) } \\
\text { 7) Multihabilidades de los operarios (porciento implantado) } \\
\text { 8) Detección automática de defectos (porciento implantado) } \\
\text { 9) Producción nivelada y mezclada (porciento implantado) } \\
\text { 10) Kaizen - mejora continua, (porciento implantado) } \\
\text { 11) Reducción de tiempo de flujo (porciento avance) } \\
\text { 12) Reducción del tiempo de entrega (porciento de avance) }\end{array}$ \\
\hline EMS & $\begin{array}{l}\text { CS } \\
\text { GIMS } \\
\text { HC } \\
\text { PAA }\end{array}$ & $\begin{array}{l}\text { 13) Existe un equipo de alto nivel para dirigir las iniciativas sustentables (SI/NO) } \\
\text { 14) Años desarrollando iniciativas sustentables (cantidad) } \\
\text { 15) Las iniciativas sustentables han permitido mejorar la productividad (Likert) } \\
\text { 16) Nivel de reducción de la transportación (porciento de avance) } \\
\text { 17) Nivel de aplicación del mapeo ambiental (porciento de avance) } \\
\text { 18) Cuenta con métricos para evaluar el desempeño sustentable (Likert) } \\
\text { 20) Se desarrollan productos medioambientalmente innovadores (Likert) } \\
\text { 21) Las iniciativas sustentables superan las regulaciones de gobierno (Likert) }\end{array}$ \\
\hline EMC & $\begin{array}{l}\text { CMC } \\
\text { GIMC } \\
\text { PEMC } \\
\text { SI }\end{array}$ & $\begin{array}{l}\text { 22) Nivel de compromiso de la administración con la mejora continua (Likert) } \\
\text { 23) Cuenta con un sistema de evaluación y seguimiento de proyectos de mejora } \\
\text { y/o sugerencias (Likert) } \\
\text { 24) Los proyectos de mejora se orientan a la seguridad de los empleados } \\
\text { (Likert) } \\
\text { 25) Personal entrenado en mejora continua (porcentaje) } \\
\text { 26) Personal que participa en proyectos de mejora (porcentaje) } \\
\text { 27) Sugerencias implantadas del total sugerido (porcentaje) }\end{array}$ \\
\hline IEORA & $\begin{array}{l}\text { IHC } \\
\text { RF } \\
\text { SE }\end{array}$ & $\begin{array}{l}\text { 28) El mejoramiento del ambiente es un motivador para adoptar iniciativas } \\
\text { sustentables (Likert) } \\
\text { 29) La mejora de la imagen pública es un motivador para adoptar iniciativas } \\
\text { sustentables (Likert) } \\
\text { 30) Seguir una directriz corporativa es un motivador para adoptar iniciativas } \\
\text { sustentables (Likert) } \\
\text { 31) Cumplir con la responsabilidad moral fue un motivador para adoptar } \\
\text { iniciativas sustentables (Likert) } \\
\text { 32) Lograr ventajas competitivas y estratégicas fue un motivador para adoptar } \\
\text { iniciativas sustentables (Likert) } \\
\text { 33) Los indicadores en materia de sustentabilidad y desempeño operacional } \\
\text { están relacionados (Likert) } \\
\text { 34) Nivel de mejora en la reducción de CO2 (porcentaje) } \\
\text { 35) La planta ayuda al cuidado del medio ambiente (Likert) } \\
\text { 36) Beneficio económico obtenido por la aplicación de iniciativas sustentables } \\
\text { (Likert) } \\
\text { 37) La planta realiza proyectos sustentables para el mejoramiento de la } \\
\text { seguridad de los empleados (Likert) } \\
\text { 38) La ejecución de iniciativas sustentables de la planta le satisfacen e } \\
\text { influencian su deseo de permanecer laborando en ella (Likert) }\end{array}$ \\
\hline
\end{tabular}

\section{RESULTADOS}

\section{Perfil de las plantas encuestadas}

El estudio se realizó en 40 plantas de diferentes giros y la manera como se integra el total es la siguiente: metal-mecánicas el $32.50 \%$ (13), autopartes el $12.50 \%$ (5), ensambles electrónicos y ensambles eléctricos el $10.00 \%$ (4) cada uno, termo-formado y cerámica $7.50 \%$ (3) cada especialidad, conversión/papel y ensamble de vehículos automotores un $5.00 \%$ (2) cada uno, aeroespacial y alimentos/bebidas un $2.50 \%$ cada uno (1) y en otras manufacturas un $5.00 \%$ (2). En cuanto al tamaño de las empresas, el $72.50 \%$ (29) son grandes y el $27.50 \%$ (11) medianas, en el caso de México de acuerdo al Instituto Nacional de Geografía 
Estadística e Informática (INEGI, 2009), las empresas de manufactura se consideran grandes cuando tienen más de 250 empleados en tanto que las medianas se encuentran en el rango de 50 a 250 trabajadores.

\section{Mediciones operacionales}

Todos los constructos en el modelo propuesto, están basados en escalas reflexivas con múltiples ítems, por lo que deben ser confirmadas su validez y Confiabilidad, así como la capacidad predictiva que tiene el modelo para predecir comportamientos de los constructos endógenos EMS, EMC e IEORA a partir del constructo exógeno EME (Coelho et al., 2012; Hair et al., 2011), en esta investigación para efectos de validar la calidad del modelo se usaron los criterios referidos por Hair et al., (2011) y Henseler et al., (2009).

\section{Estimación y resultados del modelo de medición (modelo externo)}

La estimación del modelo se realizó mediante SMARTPLS 2.0 (Ringle et al., 2005) con 40 casos empleando el algoritmo PLS y los resultados obtenidos se muestran en la figura 2.



Fig. 2: Resultados - poder explicativo $R^{2}$, betas estandarizadas y cargas - algoritmo PLS

A continuación se muestran algunas tablas exhibiendo valores no mostrados en la figura 2, que son resultantes de la corrida del algoritmo PLS, Bootstrap y Blindfolding del PLS SMART 2.0 (Ringle et al., 2005) y que se requieren para el diagnóstico y análisis completo de los resultados.

Tabla 2. Valores de AVE, confiabilidad compuesta $(\rho c)$ y alfa de Cronbach

\begin{tabular}{|l|c|c|c|}
\hline \multicolumn{1}{|c|}{ Constructo } & AVE & $\begin{array}{c}\text { Confiabilidad } \\
\text { compuesta } \rho \mathrm{c}\end{array}$ & $\begin{array}{c}\text { Alfa de } \\
\text { Cronbach }\end{array}$ \\
\hline EMC & 0.55 & 0.83 & 0.73 \\
\hline EME & 0.64 & 0.84 & 0.75 \\
\hline EMS & 0.59 & 0.88 & 0.82 \\
\hline IEORA & 0.59 & 0.85 & 0.77 \\
\hline
\end{tabular}

Tabla 3. Valores de redundancia cruzados y validados $-C V-\operatorname{Red}\left(Q^{2}\right)$ - Algoritmo blindfolding

\begin{tabular}{|l|c|}
\hline & 1- SSE/SSO CV Red $\mathrm{Q}^{2}$ \\
\hline EMC & 0.12 \\
\hline EME & 0.23 \\
\hline EMS & 0.22 \\
\hline IEORA & 0.39 \\
\hline
\end{tabular}


Tabla 4. Correlaciones entre variables latentes

\begin{tabular}{|l|c|c|c|c|}
\hline & EMC & EME & EMS & IEORA \\
\hline EMC & 1.00 & & & \\
\hline EME & 0.46 & 1.00 & & \\
\hline EMS & 0.63 & 0.62 & 1.00 & \\
\hline IEORA & 0.69 & 0.71 & 0.84 & 1.00 \\
\hline
\end{tabular}

Tabla 5. Cargas cruzadas

\begin{tabular}{|l|c|c|c|c|}
\hline INDICADOR & EMC & EME & EMS & IEORA \\
\hline CMC & 0.81 & 0.40 & 0.58 & 0.53 \\
\hline CS & 0.53 & 0.50 & 0.75 & 0.56 \\
\hline CSO & 0.60 & 0.52 & 0.71 & 0.78 \\
\hline GIMC & 0.73 & 0.39 & 0.48 & 0.62 \\
\hline GIME & 0.53 & 0.81 & 0.69 & 0.70 \\
\hline GIMS & 0.31 & 0.41 & 0.67 & 0.59 \\
\hline HC & 0.48 & 0.54 & 0.76 & 0.64 \\
\hline IHC & 0.45 & 0.49 & 0.66 & 0.72 \\
\hline PAA & 0.53 & 0.42 & 0.80 & 0.70 \\
\hline PEMC & 0.71 & 0.37 & 0.46 & 0.42 \\
\hline RF & 0.43 & 0.52 & 0.42 & 0.70 \\
\hline RFT & 0.17 & 0.78 & 0.35 & 0.46 \\
\hline RLT & 0.25 & 0.80 & 0.28 & 0.41 \\
\hline SE & 0.61 & 0.64 & 0.74 & 0.86 \\
\hline SI & 0.72 & 0.14 & 0.31 & 0.47 \\
\hline SRA & 0.56 & 0.52 & 0.84 & 0.72 \\
\hline
\end{tabular}

Tabla 6. Efectos Totales, directos e indirectos columnas (origen) y filas (destino)

\begin{tabular}{|l|c|c|c|}
\hline & EMC & EMS & IEORA \\
\hline EMC & & 0.44 & 0.47 \\
\hline EME & 0.46 & 0.62 & 0.71 \\
\hline EMS & & & 0.52 \\
\hline
\end{tabular}

Tabla 7. Tamaño de los efectos $f^{2}$

\begin{tabular}{|l|l|c|c|c|}
\hline VARIABLE & & $\mathrm{R}^{2}$ incluida & $\mathrm{R}^{2}$ excluida & $\mathrm{f}^{2}$ \\
\hline EXÓGENA & EME & 0.79 & 0.76 & 0.14 \\
\hline ENDÓGENA & EMS & 0.79 & 0.67 & 0.57 \\
\hline ENDÓGENA & EMC & 0.79 & 0.76 & 0.14 \\
\hline
\end{tabular}

En la figura 2, los valores encerrados en círculos son los coeficientes de determinación $R^{2}$, valores de 0.25 , 0.50 y 0.75 representan un poder explicativo bajo, medio y significativo de las variables latentes, las cantidades de las flechas del modelo de medición (externo) constituyen las cargas estandarizadas, ahora bien, es importante distinguir entre modelos de medición reflexivos y formativos para evaluarlos. Los modelos reflexivos deben ser evaluados en base a la confiabilidad y validez de los constructos, para estos efectos se usa la medición confiabilidad compuesta $(\rho c)$ como un estimado de la consistencia interna del constructo y de acuerdo a Hair et al., (2011) y Henseler et al., (2009) los valores de la medición deben ser mayores a 0.70 (en investigación exploratoria los valores de 0.60 a 0.70 se aceptan), en la tabla 2 se pueden observar que los valores de la confiabilidad compuesta $(\rho c)$ para los constructos EME, EMS, EMC y IEORA exceden a 0.80 indicando consistencia interna en los constructos del modelo del estudio. Cabe mencionar que a diferencia de la Alfa de Cronbach, la confiabilidad compuesta ( $\rho$ c) no asume que todos los indicadores son igualmente confiables y esto es muy conveniente para PLS-SEM, ya que esta técnica prioriza los indicadores de acuerdo a sus confiabilidad durante la estimación del modelo Hair et al., (2011) y Henseler et al., (2009).

Los indicadores del modelo de medición también deben mostrar confiabilidad, para validar este hecho se usan las cargas estandarizadas que de acuerdo a Hair et al., (2011) y Henseler et al., (2009) deben tener valores superiores a 0.70 , en la figura 2 se puede ver que las cargas de la mayor parte de los indicadores superan dicho valor con lo que se puede establecer la confiabilidad del modelo de medición y validez del instrumento. En el caso particular de la carga estandarizada de 0.67 correspondiente al indicador GIMS, este indicador no se eliminó ya que de acuerdo a (Hair et al., 2011) solo se deben excluir indicadores con cargas estandarizadas menores a 0.70 si su eliminación eleva la confiabilidad compuesta $(\rho c)$ al umbral considerado crítico. 
El diagnóstico de validez en los modelos de medición reflexivos, se enfoca en la validez convergente y la validez Discriminante. En el caso de validez convergente los valores de la varianza extraída promedio (AVE) por sus siglas en inglés deben mostrar cantidades superiores a 0.50 en todos los constructos, y se puede observar en la tabla 2 que en el caso del presente estudio los valores superan la cantidad mencionada indicando un grado suficiente de validez convergente, lo que significa que una determinada variable latente explica más de la mitad de la varianza que sus indicadores (Hair et al., 2011). Por lo que se refiere a la validez discriminante existen dos criterios complementarios de acuerdo a Hair et al., (2011) y Henseler et al., (2009), a) el criterio de Fornell-Larcker que diagnostica a nivel de constructo y b) criterio de las cargas cruzadas que diagnostica a nivel de indicadores. Para el caso del criterio de Fornell-Larcker el principio postula que una variable latente comparte más varianza con sus propios indicadores asignados que con cualquier otra variable latente, en otras palabras la AVE de cada variable latente debe ser mayor que la correlación elevada al cuadrado con cualquier otra variable latente, revisando las tablas 2 y 4 es posible observar que el criterio se cumple para la EMC, EME, EMS más no así para la relación del EMS con IEORA cuya correlación elevada al cuadrado resulta en una cantidad ligeramente mayor al AVE de IEORA. Por lo que se refiere al criterio de cargas cruzadas este criterio establece que un indicador debe tener una correlación más grande con su propia variable latente que con las otras del modelo, en la tabla 5 puede verse que este criterio es satisfecho completamente.

Con respecto a los efectos totales (directos e indirectos) se puede observar en la tabla 6 que la EMS tiene un efecto directo en el IEORA de 0.52, mientras que la EME tiene un efecto indirecto de 0.71 ya que cuenta con la contribución de la EMS y EMC 0.62 y 0.46 respectivamente, la EMC también tiene un efecto indirecto de 0.47 contando con el tributo de la EMS con 0.44. Los análisis mencionadas sirven para comprobar la validez del modelo, el poder explicativo de las variables EME, EMS, EMC sobre el IEORA y el IEORA sobre las variables dependientes RF, IHC, SE y CSO, probar hipótesis, así como para identificar hallazgos importantes de la muestra de las plantas analizadas, lo que permite generar conclusiones e implicaciones, mismas que se describen en la sección correspondiente a conclusiones.

\section{Modelo de medición (modelo interno)}

La estimación del modelo interno se realizó mediante SMARTPLS 2.0 (Ringle et al., 2005) con 40 casos empleando el algoritmo Bootstrap y los resultados obtenidos se muestran en la figura 3, las cantidades en las flechas tanto del modelo de medición (externo) como del modelo estructural (interno) representan los valores de la prueba t-student de las variables e indicadores, lo que permite reforzar las hipótesis e indicar el nivel de significancia o error $\infty$ para niveles de confianza de 95 o 99\%, así como obtener conclusiones e implicaciones acerca del modelo.

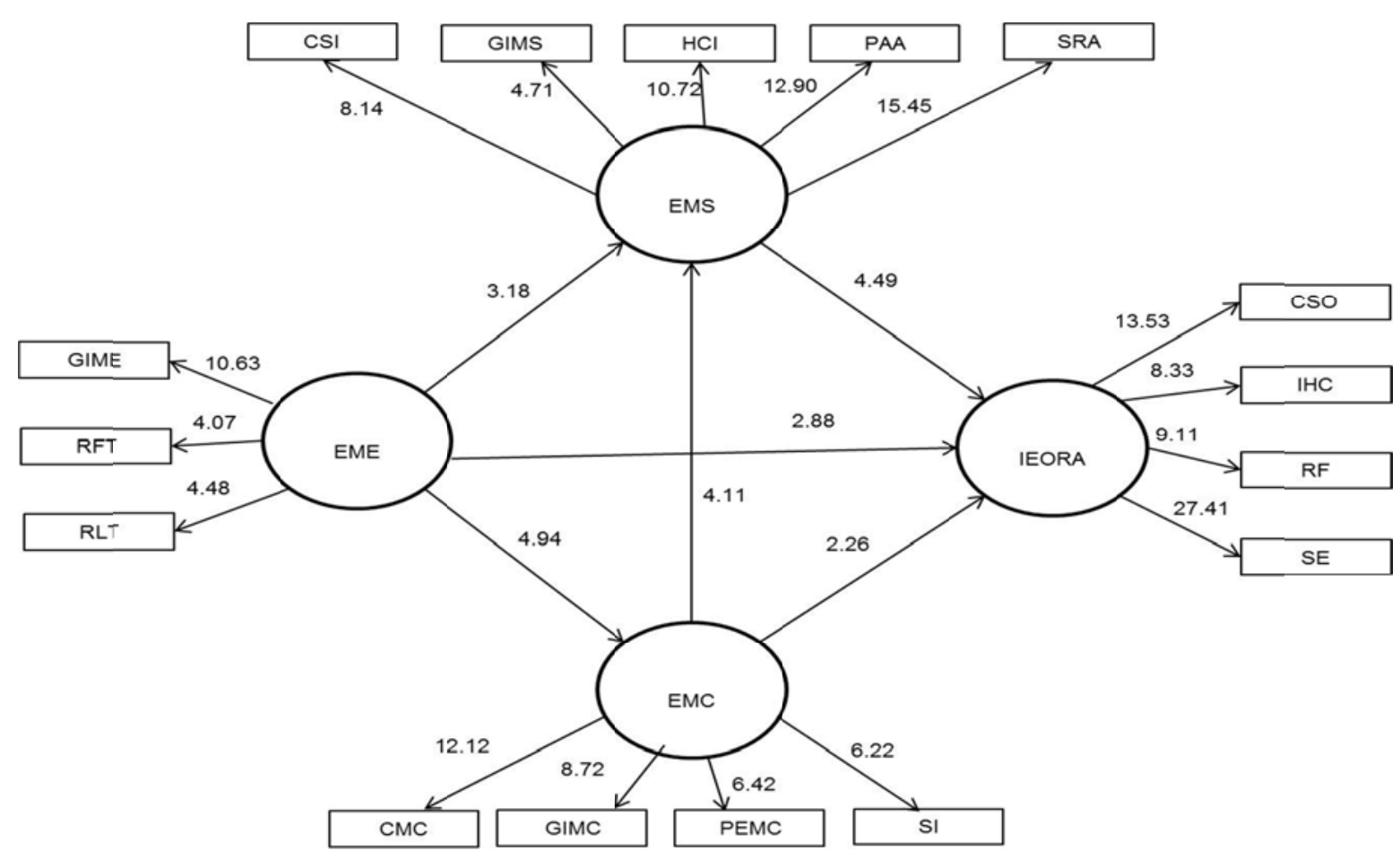

Fig. 3: Resultados mostrando los valores T de las variables e indicadores - Algoritmo Boostrapping 


\section{Estimación del modelo estructural (modelo interno)}

Es importante recordar que la modelación con ecuaciones estructurales mediante el uso de mínimos cuadrados parciales (PLS-SEM) por sus siglas en inglés no asume que los datos de los casos están normalmente distribuidos, y por lo tanto PLS aplica el procedimiento bootstrapping no paramétrico, lo que significa que realiza repetidos muestreos aleatorios con remplazo de la muestra original para crear una muestra bootstrap y con ella obtener errores estándares para pruebas de hipótesis, el proceso asume que la distribución de las muestras es una representación razonable de la distribución de la población. La muestra bootstrap permite que los coeficientes estimados mediante el algoritmo PLS-SEM sean probados para obtener su nivel de significancia Hair et al., (2011) y Henseler et al., (2009).

El criterio primario de evaluación del modelo estructural son las mediciones de la $R^{2}$ y el nivel de significancia de los path coefficients, y en virtud de que la meta de predicción de PLS-SEM es explicar la varianza de las variables endógenas, es necesario que los valores de $R^{2}$ de los constructos claves sean elevados, definir que es elevado, depende de la disciplina de la investigación de que se trate. Valores de $\mathrm{R}^{2}$ de $0.75,0.5$ o 0.25 para variables latentes endógenas en el modelo estructural se consideran sustanciales, moderados o débiles respectivamente (Hair et al., 2011). En el caso de la presente investigación en la Figura 1, se muestran valores que van de débiles en el caso de la EMC a moderados y sustanciales de las $\mathrm{R}^{2}$ en las variables latentes EMS e IEORA del modelo estructural, en particular cabe resaltar el valor de la $R^{2}$ en la variable latente endógena IEORA que exhibe un nivel sustancial de 0.79 . Los valores mencionados se obtuvieron usando el algoritmo bootstrap de SMARTPLS 2.0 con 5000 muestras (Ringle et al, 2005).

Para el diagnóstico del nivel de significancia de los path coeficients se usó nuevamente el algoritmo bootstrap y de acuerdo a Hair et al., (2011) y Henseler et al., (2009) valores críticos de $T$ son: para una

prueba de dos colas 1.65 (nivel de significancia $=0.10$ o 10\%), 1.96 (nivel de significancia $=0.05$ o $5 \%$ ) y 2.58 (nivel de significancia $=0.01$ o $1 \%$ ). En la presente investigación la Figura No. 3 muestra los valores de T para el modelo estructural y salvo el valor de T de EMC $\rightarrow$ IEORA de 2.26 que es significante en un poco más de 0.05 o sea $5 \%$, el resto de lo valores es significante en al menos 0.01 o $1 \%$. En el caso particular de EMC $\rightarrow$ IEROA, pueden explicarse las razones; existe literatura que indica que en las plantas de manufactura occidentales, la administración considera que el éxito de la manufactura esbelta consiste únicamente en implantar herramientas Lean de manera aislada y sin mayor compromiso o liderazgo de la alta administración, debido a esto dichas plantas fracasan en su viaje hacia la manufactura esbelta o bien solo consiguen silos esbeltos y beneficios marginales. Lo anterior significa en términos simples, que la manufactura esbelta en la situación mencionada no se implanta como un sistema de mejora continua (Liker et al., 2011; Koenigsaecker, 2009), por lo que respecta a los valores de T del modelo de medición (externo) estos son significantes al menos en 0.01 o $1 \%$.

En relación a la relevancia predictiva se corrió el algoritmo blindfolding de SMART PLS 2.0 (Ringle et al., 2005). Uno de los objetivos y aportaciones que el presente estudio persigue es que el modelo de la investigación (modelo IEORA) tenga capacidad predictiva del comportamiento del constructo endógeno (IEORA), con el fin de que este pueda guiar a los usuarios en sus esfuerzos de mejora al correr el modelo y obtener valores en la variable latente IEORA. Para realizar el diagnóstico de la capacidad de predecir o relevancia predictiva del modelo estructural se usó el algoritmo blindfolding del PLS-SEM para obtener la redundancia validada y cruzada (cross validated redundancy o CV Red), la medición predominante es la $Q^{2}$ que postula que el modelo estructural debe ser capaz de predecir adecuadamente cada indicador del constructo latente endógeno (Hair et al., 2011) en este estudio la variable latente IEORA.

La $Q^{2}$ se obtiene usando el algoritmo blindfolding, una técnica de reuso de muestra que omite cada dth parte de un dato y usa el estimado resultante para predecir la parte omitida. El procedimiento blindfolding se aplica únicamente a constructos latentes endógenos que tienen un modelo de medición reflexivo. Para realizar el diagnóstico de $Q^{2}$ la medida de $C V$ Red de un determinado constructo latente endógeno debe tener valores mayores a 0.00 , lo que se interpreta como la relevancia predictiva o explicativa de los constructos latentes exógenos sobre los constructos endógenos en consideración. En el caso del presente estudio se observa en la Tabla 3, que los valores de CV Red para los constructos del modelo tienen valores superiores a 0.00 , lo que confirma la relevancia predictiva de los constructos exógenos sobre los constructos endógenos, es decir que es posible predecir el comportamiento de los constructos endógenos en este caso EMS, EMC e IEORA a partir de los constructos exógenos.

En relación al tamaño del efecto o $f^{2}$, es una medición importante en el diagnóstico del modelo estructural, que se interpreta como una calibración para determinar si una variable latente tiene un efecto débil (0.02), medio (0.15) o alto (0.35) en el modelo estructural Coelho et al., (2012), Hair et al., (2011) y Henseler et al., 
(2009). En el caso de la presente investigación los valores de $f^{2}$ se muestran en la tabla 7 , y como se puede observar los efectos no son débiles, siendo de hecho alto para la EMS lo que significa un efecto importante en el modelo estructural, en general puede desprenderse de este análisis que tanto las variables latentes exógenas como las endógenas tienen un efecto relevante en el modelo estructural de la investigación.

Es importante mencionar que con mucha frecuencia los estudios acerca de ecuaciones estructurales encontrados en la literatura no agotan todos los criterios de calidad (Hair et al., 2012) mencionados en (Hair et al., 2011), esto constituye una seria omisión, ya que una medición reflexiva confiable y válida debe cumplir todos los criterios (Henseler et al., 2009), por ello en esta investigación se han incluido la totalidad de los criterios para diagnosticar modelos PLS-SEM aplicables a la situación particular.

Es importante discutir que en esta investigación se ha probado que la manufactura esbelta, la manufactura sustentable y la mejora continua tienen un efecto positivo, directo y estadísticamente significativo en la eficiencia operacional y responsabilidad ambiental, esto es consistente con lo que establece la literatura revisada y estudios previos (Ghosh, 2013; Toussaint et al., 2013; Murugesan et al., 2012; Vinohd et al., 2012; Bergmiller et al., 2011; Slaper, 2011). Sin embargo resulta de particular interés hacer notar que el estudio también muestra, que los impactos en la eficiencia operacional y responsabilidad ambiental de la mejora continua y la manufactura esbelta son menores al de la manufactura sustentable, esta situación también ha sido consignada en la literatura para el caso de las plantas de manufactura occidentales, siendo los argumentos principales, la carencia de compromiso de la alta administración, falta de capacitación y entrenamiento, carencia de constancia en el propósito, así como el hecho de que estas filosofías se implantan como esfuerzos aislado "silos", y no como un proceso de

mejora continua (Toussaint et al., 2013; Bergmiller et al., 2011; Liker et al., 2011; Reyes-Aguilar, 2002) que requiere una verdadera transformación cultural (Toussaint et al., 2013; Shook, 2010).

\section{PRUEBAS DE HIPÓTESIS}

En relación a la hipótesis correlacional, Ho., existe un grado de impacto directo y positivo similar de las variables EME, EMS y EMC en el IEORA, lo que se establece en la expresión descrita por la ecuación 7.

$\mathrm{Ho}:(E M E$, EMS, EMC $) \rightarrow$ IEORA
$\beta_{\text {EME,IEORA }} \approx \beta_{\text {EMS,IEORA }} \approx \beta_{\text {EMC,IEORA }}$

Revisando las hipótesis alternativas que se establecen en las ecuaciones 8 y 9 . Existe una diferencia significativa del nivel de impacto de las variables: EME, EMS y EMC en el IEORA.

Ha1: $\beta_{E M E, I E O R A} \neq \beta_{\text {EMS,IEORA }} \neq \beta_{\text {EMC,IEORA }} \rightarrow$ IEORA
Ha2: $\beta_{\text {EME,IEORA }}>\left(\beta_{\text {EMS, IEORA }}, \beta_{\text {EMC,IEORA }}\right) \rightarrow$ IEORA

Se rechaza la hipótesis nula, ya que las betas estandarizadas (path coefficients) son bastante diferentes para los diferentes constructos en particular para la beta de EME y EMC con el IEORA como se muestra en la figura 3, se aprueba la hipótesis alternativa $\mathrm{Ha} 1$ con los niveles de significancia ya referidos en las secciones anteriores. En los casos Ho y Ha1 la relación causal positiva de los constructos EME, EMC y EMS con el IEORA queda comprobada. Por lo que respecta a la hipótesis alternativa $\mathrm{Ha2}$, se rechaza ya que la beta estandarizada de la EME con el IEORA no es mayor que la beta estandarizada de la EMS con el IEORA aunque si ligeramente superior a la beta de EMC con el IEORA, indicando que hay un mayor grado de impacto de la EMS en el IEORA. La siguiente parte de las hipótesis derivadas del modelo gráfico, se describe a continuación. Existe un grado de influencia similar de la variable IEORA en las variables: RF, IHC, SE y CSO, lo que se indica para la hipótesis nula en la ecuación 10.

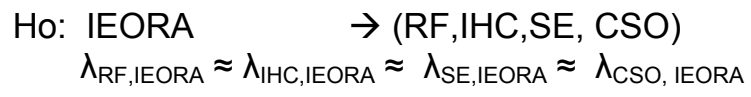

Las hipótesis alternativas están dadas de la forma siguiente. Existe una diferencia significativa en las cargas factoriales del IEORA en las variables: RF, IHC, SE, CSO, lo que queda descrito en las ecuaciones 11 y 12.

Ha3 $\lambda_{\mathrm{RF}, \mathrm{IEORA}} \neq \lambda_{\mathrm{IHC}, \mathrm{IEORA}} \neq \lambda_{\mathrm{SE}, \mathrm{IEORA}} \neq \lambda_{\mathrm{CSO}, \mathrm{IEORA}}$

Ha4: $\lambda_{\text {RF,IEORA }}>\left(\lambda_{\text {IHC,IEORA }}, \lambda_{\text {SE,IEORA }}, \lambda_{\text {CSO,IEORA }}\right)$ 
Se rechaza la hipótesis nula, ya que las $\lambda$ cargas estandarizadas son diferentes para las distintas variables RF, IHC, CSO y SE, son similares para IHC y RF, pero muy diferentes para CSO y SE, particularmente para esta último variable manifiesta que exhibe una carga muy grande en relación al indicador RF, se aprueba la hipótesis alternativa Ha3 con los niveles de significancia ya referidos en secciones anteriores.

Por lo que respecta a la hipótesis alternativa $\mathrm{Ha} 4$, se rechaza, ya que la $\lambda$ carga estandarizada de la $\mathrm{RF}$ con el IEORA, no es mayor que las $\lambda$ cargas estandarizadas de la SE y CSO con el IEORA, indicando que hay una mayor grado de impacto del IEORA sobre la SE y CSO con los niveles de significancia que ya han sido mencionados.

\section{CONCLUSIONES}

De los resultados mostrados y análisis de los mismos, se pueden extraer las siguientes conclusiones acerca de la investigación en las plantas de manufactura de Apodaca, México: 1) Los constructos manufactura esbelta, sustentable y mejora continua tienen un impacto directo, relevante positivo y estadísticamente significante en el constructo dependiente eficiencia operacional y responsabilidad ambiental, siendo la manufactura sustentable la de mayor impacto. Lo anterior significa que el modelo constituye un mecanismo empírico para medir los efectos de los constructos mencionados en la eficiencia operacional y responsabilidad ambiental; 2) Los constructos referidos en el inciso 1), empleados de manera combinada, poseen una capacidad predictiva importante del constructo dependiente eficiencia operacional y responsabilidad ambiental; 3) Modelos estadísticos complementarios, pueden ser usados para estimar desempeños en los indicadores clave de eficiencia operacional de las plantas de manufactura; 4) El modelo propuesto puede ayudar a priorizar acciones específicas de mejora continua y toma de decisiones, en la implantación de la manufactura esbelta y sustentable (Hölck et al., 2010).

Líneas de Investigación Futuras Es recomendable, extender el presente estudio a plantas medianas y grandes de otros estados industrializados de México, con el fin de hacer un análisis comparativo de los resultados. Asimismo, resulta conveniente emplear la información que proporciona la corrida computacional del modelo de ecuaciones estructurales del estudio, en específico de los scores de las variables latentes, para utilizarlos como datos de entrada en modelos de regresión u otras técnicas de estadística multivariadas, con el fin de generar modelos predictivos del desempeño en la eficiencia operacional y responsabilidad ambiental de las plantas, y prescribir acciones de mejora, para los diferentes giros de las plantas de manufactura analizadas.

\section{REFERENCIAS}

Amin, M.A y Karim, M.A. A time-based quantitative approach for selecting lean strategies for manufacturing organisations, International Journal of Production Research, 51(4), ISSN: 00207543, 1146-1167 (2013).

Arrieta, J.G., Botero, V.E. y Romano, M.J. Benchmarking sobre la manufactura esbelta (lean manufacturing) en el sector de la confección en la ciudad de Medellín, Colombia, Journal of Economics, Finance and Administrative Science (en línea), 15(28), ISSN: 2077-1886, 141-170 (2010).

Austin, D., Saleeshya, P.G. y Vamsi, N. A model to assess the lean capabilities of automotive industries, International Journal of Productivity and Quality Management, 11(2), ISSN: 1746-6482, 195 (2013).

Bergmiller, G.G. y McCright, P.R. Lean and sustainability programs: Evidence of operational synergy for lean manufacturers and logical growth toward sustainability, Review of Business Research, 11(5), ISSN: 15462609, 58-68 (2011).

Cardozo, E.R, Rodríguez, C. y Guaita, W. Las pequeñas y medianas empresas agroalimentarias en Venezuela y el desarrollo sustentable: Enfoque basado en los principios de la manufactura esbelta. Información Tecnológica (en línea), 22(5), ISSN: 0718-0764, 39-48 (2011).

Coelho, P.S. y Henseler, J. Creating Customer loyalty through service customization, European Journal of Marketing, 46(3/4), ISSN: 0309-0566, 331-356 (2012).

Cooper, R y Maskell, B. How to manage through worse-before better, MIT Sloan Management Review, summer 2008, 49(4), ISSN: 1532-9194, 58-65 (2008).

Cottyn, J., Van Landeghem, H., Stockman, K y Derammelaere, S. A method to align a manufacturing execution system with lean objectives, International Journal of Production Research, 49(14), ISSN: 00207543, 4397-4413 (2011). 
EPA. Pursuing perfection: Cases studies examining lean manufacturing strategies, pollution prevention and environmental regulatory management implications (2000),1-22, EPA Environmental Protection Agency, USA, http: www.epa.gov, acceso: 10 de Julio (2011).

Ghosh, M. Lean Manufacturing performance in indian manufacturing plants, Journal of Manufacturing Technology Management, 24(1), ISSN: 1741038X, 113-122 (2013).

Hair, J.F, Sarstedt, M. y Ringle, C.M., An assessment of the use of partial least squares structural equation modeling in marketing research (on line), Journal of the Academy of Marketing Science, 40, ISSN: 15527824, 414-433 (2012).

Hair, J.F., Ringle, C.M y Sarstedt, M. PLS-SEM: Indeed a silver bullet, Journal of Marketing Theory and Practice, 19(2) Spring 2011, ISSN: 1069-6679, 139-151 (2011).

Henseler J, Ringle C.M y Sinkovics R.R. The use of partial least squares path modeling in international marketing, Advances in International Marketing, 20, ISSN: 1474-7979, 277-319 (2009).

Hernández-Sampieri, R., Fernández, R. y Baptista, P. Metodología de la Investigación, 5ed., 243-245, Mc. Graw Hill, DF, México (2010).

Hölck, C, Ringle, C.M. y Sarstedt, M. Management of multi-purpose stadiums: Importance and performance measurement of services interfaces, International Journal Services Technology and Management, 14(2/3), ISSN: 1460-6720, 188-204 (2010).

Imai, M. Kaizen: La Clave de la Ventaja Competitiva Japonesa, 1era. Ed, CECSA, DF México (1986).

INEGI, Censos económicos 2009. Micro, pequeña, mediana y gran empresa, Instituto Nacional de Geografía Estadística e Informática, ISBN: 970-13-4739-023-61, DF México (2009).

Irastorza, V. y Fernández, X. Balance nacional de energía y su relación con el inventario nacional de emisiones (en línea), Revista Internacional de Estadística y Geografía México, 1(1), Noviembre, ISSN en trámite, 52-71 (2010), acceso: 10 de Marzo de (2013), http://rde.inegi.org.mx/revista_noviembre_2010/.

Jiang, Z., Zhang, H y Sutherland, J.W. Development of an environmental performance assessment method for manufacturing process plans, International Journal of Advanced Manufacturing Technologies (on line), (58), ISSN: 1433-3015, 783-790 (2012).

Kidwell, M. Lean Manufacturing and the environment: Ignoring the $8^{\text {th }}$ deadly waste leaves money on the table, Association for Manufacturing Excellence Target Magazine, 22(6), 13-18 (2006).

Koenigsaecker, G. Leading the Lean Enterprise Transformation, 39-77, CRC Press, New York, USA (2009).

KPMG México, Encuesta: Desarrollo sustentable en México (2009), 4-27, KPMG Cárdenas Dosal, S.C., México DF, http://kpmg.com.mx, acceso: 5 de Noviembre de (2011).

Lee, S. The impact of manufacturing practices on operational performance, Review of business research, 12(5), ISSN: 1546-2609, 184-189 (2012).

Liker, J y Convis, G. The Toyota way to lean leadership, 1-10, CRC Press, New York, USA (2011).

Millar, H.H y Rusell, S. The adoption of sustainable manufacturing practices in the Caribbean, Business Strategy and the Environment, 20, ISSN: 0964-4733, 512-526 (2011).

MIT y BCG, Findings from the 2012 sustainability and innovation global executive study and research report, MIT Sloan Management Review research report, winter, ISSN: 1532-9194, 1-13 (2013).

MIT y BCG, Sustainability: The "Embracers" seize the advantage, MIT Sloan Management Review Research Report, winter, ISSN: 1532-9194, 5-22 (2011).

MIT y BCG, The business of sustainability, MIT Sloan Management Review, Special Report, ISSN: 15329194, 3-14 (2009). 
Murugesan, T.K., Kumar, B.S. y Kumar, M.S, Competitive advantage of world class manufacturing system (WCMS) - A study of manufacturing companies in south India, European Journal of Social Sciences, 29(2), ISSN: 1450-2267, 295-311 (2012).

Pérez, J., La Rotta, D., Sánchez, K. y Madera, Y. Identificación y caracterización de las mudas de transporte, procesos, movimientos y tiempos de espera en nueve pymes manufactureras incorporando la perspectiva del nivel operativo (en línea), Ingenierae: Revista chilena de ingeniería, 19(3), ISSN: 0718-3305, 396-408 (2011).

Reyes-Aguilar, P. Manufactura delgada (lean) y seis sigma en empresas mexicanas: experiencia y reflexiones, Revista Contaduría Pública y Administración México, 205(Abril-Junio), ISSN: 0186-1042, 51-67 (2002).

Ringle, C, Wende, S y Will, A. Smart PLS 2.0 M3; Next generation path modeling software, Hamburg, Germany (2005).

Rodríguez, I. Construya la eficiencia energética, Revista Manufactura Expansión México, Feb (2011), 41-45 (2011).

Schneider Study, Execs see energy as business, moral imperative, (en línea), EEUU, Plant Engineering, (2011), 1-2, acceso: 14 de Marzo (2011), http://www.plantengineering.com/single-article/schneider-studyexecs-see-energy-as-business-moral-imperative.

Shook, J. How to change a culture: Lessons from NUMMI, Sloan Management Review, winter 2010, 51(2), ISSN: 1532-9194, 63-68 (2010).

Silveira, T.A. y Gati, A.M. Environmental value stream mapping (EVSM) as a sustainability management tool, Conference on Management of Engineering \& Technology, Portland, USA, August (2009), E-ISBN: 978-1890843-20-5, 1-34 (2009).

Slaper, T.F. The triple bottom line: What is it and how does It work?, Journal Indiana University Kelley School of Business, spring (2011), ISSN: 0007-6813, 4-8 (2011).

Tavakol, M. y Dennick, R. Making sense of Cronbach's alpha, International journal of medical education, 2, ISSN: 2042-6372, 53-55 (2011).

Toussaint. J.S. y Berry, L.L. The Promise of Lean in health care (on line), Mayo clinic proceedings, 88(1), ISSN: 1942-5546, 72-84 (2013).

Upadhye, N., Deshmukh, D.G. y Garg, S. Lean manufacturing for sustainable development, Global Business and Management Research, 2(1), ISSN: 1947-5657, 125-137 (2010).

Wills, B. Green intentions; Creating a green value stream to compete and win, 3-34, CRC Press, New York, USA (2009a).

Wills, B. The Business case for environmental sustainability (green); Achieving rapid returns from the practical integration of lean and green (2009b). HPS white paper, 1-6, acceso: 5 de Enero 2009, http://www.leanandgreensummit.com/LGBC.pdf.

Vinodh, S y Dino, J. Structural equation modeling of lean manufacturing practices, International Journal of Production Research,50(6), ISSN: 00207543, 1598-1607 (2012).

Womack, J.P, Jones, D.T y Roos, D. The machine that changed the world: The story of lean production systems $1^{\text {st }}$. Ed., Rawson Associates, 11-15, New York, USA (1990). 
